ISSN 0001-6012/2020/62/1/18-25 Acta Médica Costarricense, (C) 2020 Colegio de Médicos y Cirujanos de Costa Rica

\title{
Original
}

\section{Fatiga asociada al cáncer de mama luego de un programa de entrenamiento}

\section{(Fatigue associated with breast cancer after a training program)}

Javier Eliécer Pereira-Rodríguez, ${ }^{1}$ Devi Geesel Peñaranda-Florez, ${ }^{2}$ Ricardo Pereira-Rodríguez, ${ }^{3}$ Pedro PereiraRodríguez, ${ }^{4}$ Ximena Velásquez-Badillo, ${ }^{5}$ Yair Alberto Cañizares-Pérez ${ }^{6}$

\section{Resumen}

Antecedente y objetivo: el cáncer es una de las enfermedades que genera mayor mortalidad en el mundo. Los programas de rehabilitación basados en ejercicios se muestran efectivos y seguros para mitigar los efectos del cáncer. El objetivo fue determinar los cambios de la fatiga asociada al cáncer de mama luego de un programa de entrenamiento de alta intensidad (HIIT), o continuo a intensidad moderada (MICT).

Métodos: ensayo clínico aleatorizado con 236 pacientes con cáncer de mama en estadio II, distribuidos en 3 grupos (MICT, HIIT y grupo control), a quienes se les aplicó la escala FACTFatigue Scale, test de calidad de vida, ecocardiograma, prueba de esfuerzo, tolerancia y percepción al ejercicio mediante la prueba de caminata de los 6 minutos y escala de Borg, respectivamente. El programa de entrenamiento tuvo una duración de 36 sesiones de 70 minutos, 3 veces por semana.

Resultados: luego de comparar los respectivos grupos, se evidenció cambios significativos en todas las variables de los grupos HIIT y MICT ( $p=<0,05 \%)$ frente al grupo control. Además, hubo una mejoría posentrenamiento, de la fatiga asociada al cáncer de mama en el grupo HIT $(20,4 \pm 5,6$ vs $5,1 \pm 3,6)$ y $\operatorname{MICT}(18.6 \pm 9.5$ vs $8.0 \pm 4.2)$.

Conclusiones: estos tipos de entrenamiento mejoraron la tolerancia al ejercicio, fuerza, vo2 y sobre todo, la fatiga asociada al cáncer y la calidad de vida de las pacientes. Y el grupo control de atención habitual sin entrenamiento físico o ejercicio supervisado, no presentó cambios significativos ni mejoras en la fatiga asociada al cáncer de mama.

Trial registration: Clinicaltrials.gov NCT03915288

Descriptores: cáncer, fatiga, ejercicio aeróbico, entrenamiento de alta intensidad, fuerza.

\footnotetext{
Afiliación de los autores: 'Hospital Ángeles Lomas Huixquilucan y Universidad Tolteca, Puebla, México. ${ }^{2}$ Consultorio Independiente Privado, Puebla, México. ${ }^{3}$ Fundación Universitaria de Ciencias de la Salud. BogotáColombia. ${ }^{4}$ Universidad Rafael Núñez, Cúcuta-Colombia. ${ }^{5}$ Clínica San José. Cúcuta-Colombia. ${ }^{6}$ Universidad de Santander. Cúcuta-Colombia. Grupo de investigación Aletheia

Conflicto de interés: los autores declaran no tener. No hubo alguna financiación externa. 凶jepr87@hotmail.com
} 
Results: after comparing the respective groups, significant changes were evident in all the variables of the HIIT and MICT groups ( $p=<0.05 \%$ ) compared to the control group. Also, there was a postworkout improvement of fatigue associated with breast cancer in the HIIT group ( $20.4 \pm 5.6$ vs. 5.1 $\pm 3.6)$ and MICT $(18 \mathrm{~m} 6 \pm 9 \mathrm{~m} 5$ vs. $8 \mathrm{~m} 0 \pm 4 \mathrm{~m} 2)$.

Conclusions: these types of training improved exercise tolerance, strength, VO2 and, above all, cancer-associated fatigue and patients quality of life. The usual care control group without physical training or supervised exercise did not show significant changes or improvements in fatigue associated with breast cancer. Trial registration: Clinicaltrials.gov NCT03915288

Trial registration: Clinicaltrials.gov NCT03915288.

Keywords: Cancer, fatigue, aerobic exercise, high intensity training, strength.

Fecha recibido: 19 de junio 2019 Fecha aprobado: 21 de noviembre 2019

El cáncer (CA) es la segunda causa de muerte en el mundo; para 2015 se notificaron 8,8 millones de muertes por esta enfermedad. El de pulmón, hígado, colón y recto, estómago y mama, son los principales tipos de cáncer con mayor mortalidad en todo el mundo. ${ }^{1}$

Cáncer, tumores o neoplasias malignas, son términos que se asignan a un conjunto de enfermedades que afectan a múltiples órganos blancos. En esta enfermedad ocurre un crecimiento acelerado y múltiple de células anormales, que sobrepasan los espacios normales e invaden otros sitios donde no suelen presentarse, colonizando tejidos y órganos, es decir, lo que comúnmente se conoce como metástasis. ${ }^{2}$

En las Américas, cada año se diagnostican más de 462 mil mujeres con CA de mamá, y se estima que 100 mil mueren por esta enfermedad. Se prevé que para 2030 los casos de cáncer mamario aumentarán hasta un $34 \%$ en estas regiones. Se diagnostican más casos de neoplasias mamarias en menores de 65 años en las Américas, que en los Estados Unidos y Canadá, donde son más comúnes cuanta más edad.,3 Por 10 tanto, un diagnóstico oportuno y precoz reduce significativamente la mortalidad en pacientes con cáncer mamario. Cada vez la esperanza de vida es mayor en pacientes oncológicos con CA de mama, partiendo del hecho de donde las mujeres tienen mayor probabilidad de supevivencia que los hombres. Resalta que algunas de las complicaciones más comunes en el tratamiento para el cáncer incluyen alteraciones musculoesqueléticas, cardiovasculares y pulmonares, tales como: infección, diarrea, dolor, linfedema, náuseas, fatiga, osteopenia.., 6

La fatiga es una de las manifestaciones más comunes en el tratamiento para el manejo del cáncer, y aumenta la limitación y la discapacidad progresiva. Se ha sugerido el ejercicio como parte de la intervención terapéutica en el manejo paliativo del cáncer. De esta manera, los beneficios del ejercicio en el CA varían dependiendo del momento del tratamiento, tipo y estadio, como también de la actividad física o no de los pacientes en tratamiento. ${ }^{7}$

El ejercicio físico en pacientes oncológicos, durante o a partir del tratamiento terapéutico, previene la disminución de la función cardiovascular y cardiorrespiratoria, mejora la composición corporal, inmunológica, la fuerza muscular y flexibilidad, la imagen corporal, la autoestima y el estado de ánimo. ${ }^{8}$ A nivel vascular, el ejercicio promueve el estrés de cizallamiento vascular y reduce el estrés oxidativo, lo que estimula la función endotelial. El entrenamiento en intervalos de alta intensidad (HIIT) es una propuesta que busca aumentar la intensidad del ejercicio a partir de incrementos sobre el esfuerzo con períodos de recuperación activa. Además, diferentes estudios ${ }^{9-11}$ han demostrado los beneficios positivos de este programa de entrenamiento en pacientes con cáncer, al punto de que mejoran la función física, la calidad de vida y las actividades diarias. Así, el entrenamiento continuo de intensidad moderada (MICT, por sus siglas en inglés), ha mostrado beneficios, al igual que el HIIT, pero de una manera menos riesgosa, al tratarse de una intervención basada en ejercicio aeróbico a una intensidad moderada. ${ }^{12,13}$

Dicho lo anterior, surge como pregunta de investigación: ¿cuáles son los cambios de la fatiga asociada al cáncer de mama luego de un programa entrenamiento de alta intensidad (HIIT), o continuo a intensidad moderada (MICT), en pacientes con cáncer de mama en estadio II? El objetivo principal de la presente investigación es determinar los cambios de la fatiga asociada al cáncer de mama, luego de un programa de entrenamiento de alta intensidad (HIT), o un entrenamiento continuo a intensidad moderada (MICT).

\section{Métodos}

Esta investigación fue un ensayo clínico aleatorizado, con una muestra de 1573 pacientes con cáncer, y después de los criterios de inclusión y exclusión, 216 pacientes con cáncer de mama en estadio II, finalmente iniciaron la investigación, organizados en 3 grupos: el grupo MICT (Grupo experimental 1), el grupo HIIT (Grupo experimental 2) y el grupo de consideraciones usuales (Grupo control) (Figura 1). Se hizo un muestreo probabilístico básico por medio de una tabla de números aleatorios, cuyo orden fue aleatorio y cegado a través del programa Microsoft Excel 16.0, con resultado de 80 participantes para el grupo experimental 1, 70 en el grupo experimental 2 y 66 en el 
grupo control (recomendaciones verbales sobre ejercicio, dieta, seguimiento vía llamada telefónica y realización de actividades de la vida diaria).

El artículo es producto del macroproyecto randomizado de ONCO-EXE TRIAL, registrado en el sistema de registro y resultados de protocolo ClinicalTrials.gov de la National Library of Medicine (NLM), the National Institutes of Health (NIH) y la Food and Drug Administration (FDA): NCT03915288. El periodo de ejecución fue de 3 años y 4 meses (noviembre 2015 - febrero 2019) para obtener los siguientes atributos:

\section{Características de los participantes}

Los pacientes tenían particularidades semejantes desde el punto de vista de: etapa del cáncer, fracción de eyección, clase funcional, porcentaje muscular, grasa e IMC (índice de masa corporal), circunferencia abdominal, sobrepeso, obesidad, prevalencia de diabetes, hipertensión arterial (HTA), factores de riesgo cardiovascular. También, todos los individuos mostraron "riesgo alto", según la estratificación propuesta por la Asociación Americana de Rehabilitación Cardiopulmonar.

\section{Criterios de inclusión y exclusión}

Los individuos debían tener cáncer en estadio II, ser mayores de 18 años, querer asistir a un programa de entrenamiento y

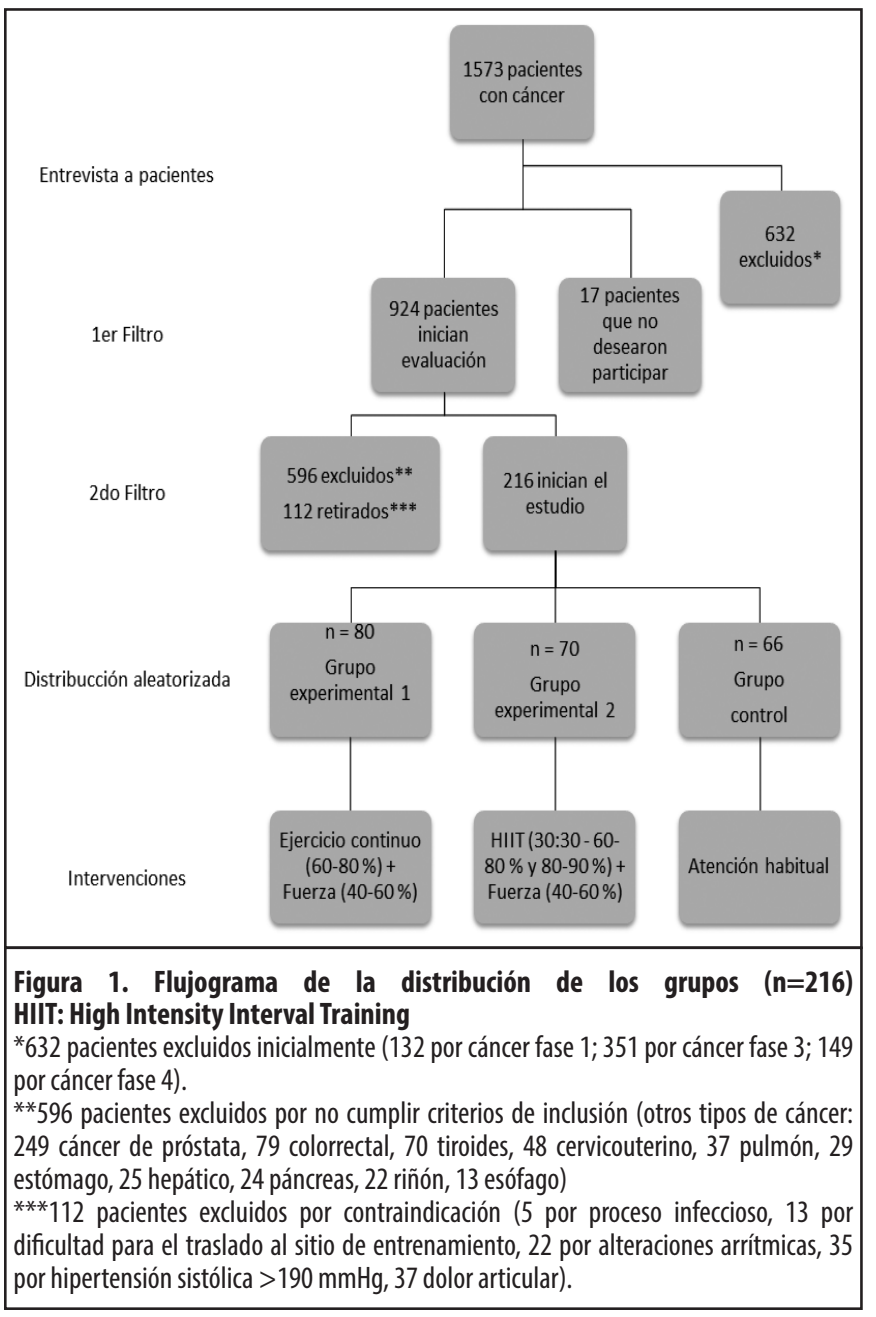

firmar un consentimiento informado protegido por el comité de ética e investigación de la institución. De la misma manera, fue obligatorio tener una fracción de eyección mayor al 35 \%, sin dificultad para realizar los cuestionarios, pruebas y las medidas requeridas por la investigación. Se evitaron los pacientes con dolor intenso en los miembros inferiores, angina inestable, frecuencia cardíaca $>120 \mathrm{lpm}$ (latidos por minuto) en reposo, presión arterial sistólica >190 mmHg, diastólica >120 mmHg, y los pacientes con cáncer en otro estadio que no fuera el II.

\section{Metodología a ciegas}

Se realizó un estudio clínico simple a ciegas, en el que los pacientes fueron evaluados inicialmente por un profesional no investigador (médico oncólogo del Servicio de Oncología). Luego, los pacientes se ingresaron a una base de datos en Microsoft Excel 16.0, exclusivamente con un número de identificación que permitió el cegamiento de los autores. Asi como iban ingresando al ensayo clínico aleatorizado, el participante era ubicado de manera aleatoria en uno de los tres grupos (MICT-HIIT-GC), conforme el computador generaba la secuencia de ubicación de grupo. Todos los participantes y profesionales, incluyendo a los investigadores, fueron cegados a lo largo del protocolo.

Para los grupos 1 y 2 (MICT-HIIT), los investigadores prepararon los cuestionarios y las pruebas sin que ellos supieran la asignación de cada paciente. Para el grupo experimental 1 y 2, los autores evaluadores fueron D. P-F. - P. P-R. y R. P-R. - Y. C-P., respectivamente.

Después de las pruebas, se sugirió a los participantes que se acercaran al autor principal (J. P-R) para informarles su horario y la fecha de inicio del programa de capacitación. Debe enfatizarse que, desde los exámenes iniciales hasta el final del programa de entrenamiento, los autores no establecieron una conversación sobre el tema con los participantes o los terapeutas. Exclusivamente, los autores realizaron cuestionarios y pruebas pre y postentrenamiento. Y solo el autor J. P-R., sostuvo reuniones periódicas con los fisioterapeutas entrenadores para conocer y armonizar el entrenamiento de cada grupo, pero no para intimar con los participantes o revisar sus datos.

Después del programa de entrenamiento, de nuevo se realizaron pruebas y exámenes a los pacientes de cada grupo para calcular los cambios posteriores al entrenamiento. Teniendo en cuenta la información recopilada antes y después, los análisis estadísticos para el grupo experimental 1 se llevaron a cabo por D. P-F. y R. P-R.; para el grupo experimental 2, por P. P-R. y X. V-B. y para el grupo control, por los autores J. P-R. y Y. C-P. Una vez que las diferentes variables se estudiaron a ciegas, todos los autores fueron informados sobre los grupos con sus pacientes y los resultados para generar las conclusiones.

\section{Medidas antropométricas}

Para todos los participantes se obtuvieron los siguientes datos: antecedentes familiares y personales, utilizando un cuestionario de creación propia, y medidas antropométricas 
(peso, talla, índice de masa corporal, circunferencia abdominal, porcentaje de grasa y músculo), con técnicas estandarizadas en la población mexicana.

El peso, el porcentaje de grasa y el músculo se obtuvieron utilizando la balanza digital Tezzio TB-30037 calibrada y ubicada en una superficie plana y estable, utilizando las indicaciones del manual del usuario. Por otro lado, la talla se obtuvo con el Adult Acrylic Halter Wall Kramer 2104. Con estas variables se determinó el IMC en $\mathrm{kg} / \mathrm{m}-1$. Luego, con una cinta métrica y una precisión de $1 \mathrm{~mm}$, se recogió la medida de la circunferencia abdominal, a partir de la referencia anatómica descrita por Frisancho. ${ }^{14}$

\section{Signos vitales}

La frecuencia cardíaca fue detectada por el sistema Polar Multisport RS800CX, y la respiratoria, al igual que la presión arterial sistólica y diastólica, manualmente, mientras que la saturación de oxígeno se obtuvo con un oxímetro portátil Nellcor Puritan Bennett. De manera similar, la disnea percibida y el esfuerzo se evaluaron utilizando la escala de Borg modificada. ${ }^{15}$

\section{Función cardiovascular}

Cada participante de la investigación fue sometido a una ecocardiografía 2-D (bidimensional) antes y después del programa de entrenamiento, para revisar las diferentes estructuras anatómicas, presiones, fracción de eyección (FEVI) como también su respectiva movilidad. La mencionada valoración, además de arrojar datos importantes sobre 10 mencionado, permitió clasificar a los pacientes en falla cardiaca con fracción de eyección reducida o preservada, y así determinar su participación. Además, se clasificaron según las 4 clases (I, II, III y IV) de la NYHA con base en las limitaciones de la actividad física del paciente, causadas por síntomas cardíacos.

\section{Prueba de esfuerzo y caminata de 6 minutos}

La Declaración ATS Statement: Guidelines for the sixminute walk test of the American Thoracic Society ${ }^{16,17}$ fue la guía para la realización de la prueba de caminata de los 6 minutos. Luego, el participante debía volver al día siguiente para una prueba de esfuerzo de acuerdo con el protocolo de Naughton, recomendado en pacientes de alto riesgo y cuya velocidad e inclinación en la banda sin fin es por estadios de 2 minutos. Para estas pruebas, se le dijo al paciente que debía evitar fumar, tomar bebidas, o cualquier tipo de medicamento que pudiera alterar sus signos vitales o el rendimiento durante la prueba.

\section{Fuerza}

La fuerza fue valorada mediante dinamometría con el Hand Grip CAMRY Electronic hand dynamometer model EH101. Para esta prueba se requirió cumplir con la siguiente técnica: sujeto de pie o sentado en posición cómoda y sin apoyabrazos. Hombros aducidos y sin rotación. Codo flexionado a $90^{\circ}$. Antebrazo y muñeca en posición neutra. Se mide la fuerza de prensión en la mano hábil y se registran tres determinaciones consecutivas, respetando el tiempo de recuperación muscular, que es de aproximadamente un minuto. Por otro lado, para conocer el peso inicial del entrenamiento de fuerza para las extremidades superiores (MMSS) e inferiores (MMII), se consideró apropiada la prueba de una repetición máxima (1RM) con una extensión completa del grupo muscular utilizado, sin sustituciones musculares.

\section{Fatiga}

La fatiga se valoró mediante la FACT-Fatigue Scale (Escala de Fatiga de la Evaluación Funcional de la Terapia del Cáncer), que es un inventario de 13 items que evalúa la gravedad de la fatiga asociada al cáncer (FAC) en la última semana, con una escala de 0 a 4, y que con puntuaciones más altas refleja una FAC más baja. ${ }^{18}$

\section{Calidad de vida}

Para este apartado se utilizó el cuestionario EORTC OLO C-30 (European Organisation for Research and Treatment of Cancer quality of Life Questionnaire Core 30), ${ }^{19}$ con su respectiva interpretación, siguiendo la guía del manual de la European Organisation for Research and Treatment of Cancer. ${ }^{20}$ La prueba fue solicitada directamente (por J. P-R) en español, a los autores principales EORTC Quality of Life Group website, quienes aprobaron su uso en la investigación.

\section{Intervenciones por grupo}

Durante las respectivas intervenciones, los participantes estuvieron monitoreados por un Polar Multisport RS800CX, oximetría y seguimiento mediante la escala de Borg, con el fin de evitar sobrepasar la intensidad de entrenamiento $(60-80 \%$ frecuencia cardíaca máxima (FCM), 6 a 8 Borg). La inclinación, resistencia o velocidad de los ejercicios fueron asignadas según los parámetros indicativos ( $\mathrm{FCM}, \mathrm{Vo}_{2}$, Borg) para intensidad moderada.

\section{Grupo experimental $1(n=80)$}

El programa de entrenamiento tuvo una duración de 36 semanas, con asistencia de 3 veces a la semana, con 70 minutos por intervención, en donde 10 minutos eran de calentamiento (ejercicios respiratorios, caminata, estiramientos), 30 minutos de entrenamiento aeróbico continuo a intensidad moderada (60-80 \% FCM), 20 minutos de entrenamiento de fuerza (4060 \% fuerza máxima) con mancuernas y theraban de manera progresiva, y los últimos 10 minutos, para el enfriamiento (ejercicios de coordinación, equilibrio, caminata y ejercicios respiratorios). Referente al MICT, este fue realizado con caminata rápida o trote en banda sin fin, con el piso inclinado para alcanzar la intensidad deseada, 10 mismo que en bicicleta, remo y elíptica.

\section{Grupo experimental $2(n=70)$}

Este grupo posee las mismas características del grupo experimental 1; las fases de calentamiento, fuerza y enfriamiento 
fueron idénticas a la del GE1. La diferencia consistió en una intensidad de entrenamiento intervalica. Es decir, los pacientes intervenidos durante los 30 minutos de ejercicio aeróbico, siguieron un protocolo creado por el autor principal (J.P-R) para este grupo experimental, que denominamos 30-30: 30 segundos a intensidad moderada (60-80\% FCM) y 30 segundos a intensidad alta (80-90\%).

\section{Grupo control $(n=66)$}

Este grupo no realizó ejercicio supervisado, ni se le otorgaron recetas de ejercicio, ni programas formales de intervención. Solo se continuó con la atención habitual y se le dieron indicaciones para mantener un estilo de vida saludable, basado en una nutrición adecuada y actividades habituales. En las instituciones adonde asistían los pacientes de los 3 grupos, no

\begin{tabular}{|c|c|c|c|}
\hline \multicolumn{4}{|c|}{ Cuadro 1. Características iniciales de la población de estudio $(n=216)$} \\
\hline Características & $\begin{array}{c}\text { Grupo } \\
\text { experimental } 1 \\
n=80\end{array}$ & $\begin{array}{l}\text { Grupo experimental } 2 \\
n=70\end{array}$ & $\begin{array}{l}\text { Grupo } \\
\text { control } \\
n=66\end{array}$ \\
\hline Sexo & $\mathrm{H}: 0 \quad \mathrm{M}: 80$ & $\mathrm{H}: 0 \quad \mathrm{M}: 70$ & $\mathrm{H}: 0 \quad \mathrm{M}: 66$ \\
\hline Edad (Años) & $51 \pm 4$ & $55 \pm 5$ & $53 \pm 7$ \\
\hline Fracción de eyección (\%) & $51 \pm 4,5$ & $45 \pm 5,3$ & $48 \pm 4,1$ \\
\hline Altura (m) & $1,50 \pm 10,2$ & $1,53 \pm 7$ & $1,53 \pm 3,4$ \\
\hline Peso $(\mathrm{kg})$ & $79 \pm 12,9$ & $83 \pm 10,5$ & $80 \pm 8,1$ \\
\hline IMC (Peso/Talla2) & $29 \pm 5,4$ & $31 \pm 3,9$ & $30 \pm 4,2$ \\
\hline Circunferencia abdominal (cm) & $90 \pm 5,2$ & $93 \pm 3,5$ & $86 \pm 6,9$ \\
\hline Porcentaje graso (\%) & $26 \pm 7,4$ & $28 \pm 4,4$ & $27 \pm 6,3$ \\
\hline Porcentaje muscular (\%) & $29 \pm 15,6$ & $32 \pm 8,1$ & $30 \pm 12,8$ \\
\hline Fuerza (Kg) & $24,5 \pm 10,5$ & $27,4 \pm 5,7$ & $29,7 \pm 6,3$ \\
\hline Vo2 estimado (Ml/kg/o2/min) & $8,3 \pm 3,2$ & $8,5 \pm 8,3$ & $8,9 \pm 5,7$ \\
\hline METs (O2/kg x min) & $2,3 \pm 0,9$ & $2,4 \pm 2,3$ & $2,5 \pm 1,6$ \\
\hline Fatiga (FACT-F) & $18,6 \pm 9,5$ & $20,4 \pm 5,6$ & $16,6 \pm 5,5$ \\
\hline Calidad de vida & $60,7 \pm 16,7$ & $58,6 \pm 17,0$ & $64,5 \pm 12,9$ \\
\hline Distancia recorrida (m) & $231 \pm 28$ & $253 \pm 10,8$ & $279 \pm 18,6$ \\
\hline FCM en prueba de esfuerzo (Ipm) & $156 \pm 6$ & $152 \pm 12$ & $155 \pm 7$ \\
\hline Sobrepeso y/u Obesidad (\%) & $81 \%$ & $85 \%$ & $88 \%$ \\
\hline Obesidad abdominal (\%) & $84 \%$ & $88 \%$ & $79 \%$ \\
\hline Dislipidemia (\%) & $34 \%$ & $45 \%$ & $27 \%$ \\
\hline Hipertensión arterial (\%) & $56 \%$ & $41 \%$ & $35 \%$ \\
\hline Diabetes (\%) & $20 \%$ & $13 \%$ & $7 \%$ \\
\hline Enfermedad renal (\%) & $5 \%$ & $3 \%$ & $6 \%$ \\
\hline Sedentarismo* (\%) & $90 \%$ & $91 \%$ & $87 \%$ \\
\hline Tabaquismo (\%) & $54 \%$ & $31 \%$ & $25 \%$ \\
\hline Alcoholismo (\%) & $2 \%$ & $9 \%$ & $1 \%$ \\
\hline Ingesta de comida inadecuada (\%) & $14 \%$ & $16 \%$ & $10 \%$ \\
\hline Historial de IAM (\%) & $6 \%$ & $4 \%$ & $9 \%$ \\
\hline Sexo femenino (\%) & $100 \%$ & $100 \%$ & $100 \%$ \\
\hline
\end{tabular}


Fatiga asociada al cáncer de mama / Pereira-Rodríguez et al

se cuenta con un área de entrenamiento físico supervisado por un profesional. Por ende, no se excluyó o negó el protocolo de intervención a los participantes del grupo control, simplemente se dio continuidad y supervisión con fines investigativos, a su proceso y tratamiento. Además, ninguno de los 3 grupos detuvo su tratamiento médico ni el de los otros profesionales que conforman el equipo interdisciplinario.

\section{Consideraciones éticas}

El diseño y desarrollo de la investigación se llevó a cabo bajo las consideraciones éticas de la Declaración de Helsinki, firma de consentimiento informado y con la autorización de los pacientes, gerentes, coordinadores y comité de ética de la institución.

\section{Análisis estadistico}

Luego de crear una base de datos en Microsoft Excel 16.0, se obtuvieron las estadísticas descriptivas. La normalidad de los datos se evaluó mediante la prueba de Kolmogorov-Smirnov y la indicación de especificidad fue evidente para todos los análisis. Con la prueba de Tukey, se utilizó el análisis de varianza ANOVA (Análisis de varianza de una vía) y, posteriormente, las pruebas post hoc para evaluar las características de los diferentes grupos de edad, género y antropometría. En todos los casos, se estableció un nivel de significación del $5 \%(p<0,05)$ y todo lo realizado se llevó a cabo en el programa Stata.

\section{Resultados}

El presente ensayo clínico aleatorizado inició con una muestra de 1573 pacientes con cáncer, y luego de los filtros se contó con la participación de 216 mujeres con cáncer de mama en estadio II.

En cuanto al nivel académico de las participantes, el 46,2 $\%(n=100)$ había completado solo la escuela primaria, el 13,9 $\%(n=30)$ estudió hasta la educación secundaria, el 17,1 \% $(n=37)$ el nivel universitario y el 22,6 \% $(n=49)$ no estudió. Los tratamientos oncológicos realizados fueron: cirugía (14 \%), radioterapia (70\%), quimioterapia (60\%) y terapia hormonal (43\%). Los factores de riesgo más prevalentes en la población de estudio fueron: sedentarismo, sobrepeso y obesidad, obesidad abdominal, hipertensión arterial, tabaquismo, dislipidemia, diabetes mellitus, dieta inadecuada, enfermedad renal y otros más (Cuadro 1).

\begin{tabular}{|c|c|c|c|c|c|c|c|c|c|}
\hline \multirow[b]{3}{*}{ Variables } & \multicolumn{6}{|c|}{ Cuadro 2. Análisis de cambios postentrenamiento $(n=216)$} & \multirow{3}{*}{$\begin{array}{c}\text { GE } 1 \\
\text { Vs } \\
\text { GE } 2 \\
\text { Valor } p\end{array}$} & \multirow{3}{*}{$\begin{array}{c}\text { GE } 1 \\
\text { Vs } \\
\text { GC } \\
\text { Valor } p\end{array}$} & \multirow{3}{*}{$\begin{array}{c}\text { GE } 2 \\
\text { Vs } \\
\text { GC } \\
\text { Valor } p\end{array}$} \\
\hline & \multicolumn{2}{|c|}{$\begin{array}{l}\text { Grupo experimental } 1 \\
\qquad n=80\end{array}$} & \multicolumn{2}{|c|}{$\begin{array}{l}\text { Grupo experimental } 2 \\
\qquad n=70\end{array}$} & \multicolumn{2}{|c|}{$\begin{array}{l}\text { Grupo control } \\
n=66\end{array}$} & & & \\
\hline & Pre & Pos & Pre & Pos & Pre & Pos & & & \\
\hline $\begin{array}{l}\text { Fracción de eyección } \\
(\%)\end{array}$ & $51 \pm 4,5$ & $52 \pm 5,4$ & $45 \pm 5,3$ & $49 \pm 3,2$ & $48 \pm 4,1$ & $48 \pm 4,4$ & 0,003 & 0,000 & 0,000 \\
\hline Peso (kg) & $79 \pm 12,9$ & $74 \pm 3,2$ & $83 \pm 10,5$ & $75 \pm 4,5$ & $80 \pm 8,1$ & $81 \pm 6,4$ & 0,001 & 0,000 & 0,000 \\
\hline $\begin{array}{l}\text { Índice de masa } \\
\text { corporal }\end{array}$ & $29 \pm 5,4$ & $24 \pm 2,8$ & $31 \pm 3,9$ & $24 \pm 1,3$ & $30 \pm 4,2$ & $30 \pm 7,1$ & 0,001 & 0,002 & 0,001 \\
\hline $\begin{array}{l}\text { Circunferencia } \\
\text { abdominal (cm) }\end{array}$ & $90 \pm 5,2$ & $85 \pm 6,3$ & $93 \pm 3,5$ & $80 \pm 5,0$ & $86 \pm 6,9$ & $86 \pm 4,9$ & 0,000 & 0,001 & 0,000 \\
\hline Porcentaje graso (\%) & $26 \pm 7,4$ & $21 \pm 2,9$ & $28 \pm 4,4$ & $20 \pm 3,5$ & $27 \pm 6,3$ & $28 \pm 8,2$ & 0,001 & 0,002 & 0,001 \\
\hline $\begin{array}{l}\text { Porcentaje muscular } \\
(\%)\end{array}$ & $29 \pm 15,6$ & $32 \pm 3,5$ & $32 \pm 8,1$ & $38 \pm 6,7$ & $30 \pm 12,8$ & $31 \pm 8,4$ & 0,000 & 0,001 & 0,001 \\
\hline Fuerza (Kg) & $24,5 \pm 10,5$ & $29,6 \pm 3,6$ & $27,4 \pm 5,7$ & $37 \pm 5,1$ & $29,7 \pm 6,3$ & $29,5 \pm 7,7$ & 0,001 & 0,002 & 0,001 \\
\hline $\begin{array}{l}\text { Vo2 estimado (Ml/kg/ } \\
\text { o2/min) }\end{array}$ & $8,3 \pm 3,2$ & $13,1 \pm 5,8$ & $8,5 \pm 8,3$ & $16,7 \pm 5,5$ & $8,9 \pm 5,7$ & $8,8 \pm 4,0$ & 0,002 & 0,001 & 0,001 \\
\hline MET (O2/kg x min) & $2,3 \pm 0,9$ & $3,7 \pm 1,6$ & $2,4 \pm 2,3$ & $4,7 \pm 1,5$ & $2,5 \pm 1,6$ & $2,4 \pm 1,1$ & 0,001 & 0,000 & 0,003 \\
\hline Fatiga (FACT-F) & $18,6 \pm 9,5$ & $8,0 \pm 4,2$ & $20,4 \pm 5,6$ & $5,1 \pm 3,6$ & $16,6 \pm 5,5$ & $16,9 \pm 4,6$ & 0,012 & 0,006 & 0,000 \\
\hline Calidad de vida & $60,7 \pm 16,7$ & $68,7 \pm 13,6$ & $58,6 \pm 17,0$ & $125 \pm 11,8$ & $64,5 \pm 12,9$ & $63,3 \pm 13,4$ & 0,005 & $<0,001$ & 0,002 \\
\hline Disnea post (TC6M) & $8 \pm 4,4$ & $5 \pm 1,0$ & $9 \pm 4,5$ & $4 \pm 1,6$ & $8 \pm 3,2$ & $8 \pm 2,3$ & 0,000 & 0,000 & 0,001 \\
\hline Fatiga post (TC6M) & $8 \pm 2,5$ & $5 \pm 2,3$ & $8 \pm 1.4$ & $3 \pm 1,7$ & $8 \pm 2,3$ & $8 \pm 2,6$ & 0,003 & 0,002 & 0,001 \\
\hline Distancia recorrida (m) & $231 \pm 28$ & $284 \pm 41$ & $253 \pm 10,8$ & $351 \pm 13$ & $279 \pm 18,6$ & $280 \pm 10,7$ & 0,000 & 0,001 & 0,000 \\
\hline FCM (lpm) & $156 \pm 6$ & $165 \pm 6$ & $152 \pm 12$ & $169 \pm 11$ & $155 \pm 7$ & $155 \pm 5$ & 0,012 & 0,000 & 0,001 \\
\hline
\end{tabular}


Por otro lado, al comparar el porcentaje de músculo (GE1: $29 \pm 15,6$ vs $32 \pm 3,5$; GE2: $32 \pm 8,1$ vs $38 \pm 6,7$; GC: $30 \pm 12,8$ vs $31 \pm 8,4$ ), grasa (GE1: $26 \pm 7,4$ vs $21 \pm 2,9$; GE2: $28 \pm 4,4$ vs $20 \pm 3,5$; GC: $30 \pm 12,8$ vs $28 \pm 8,2$ ) e IMC (GE1: $29 \pm 5,4$ vs $24 \pm 2,8$; GE2: $31 \pm 3,9$ vs $24 \pm 1,3$; GC: $30 \pm 4,2$ vs $30 \pm 7,1$ ) y el consumo máximo de oxígeno (VO2) (GE1: 8,3 $\pm 3,2$ vs 13,1 $\pm 5,8$; GE2: $8,5 \pm 8,3$ vs $16,7 \pm 5,5$; GC: $8,9 \pm 5,7$ vs $8,8 \pm 4,0$ ), como un indicador de tolerancia al ejercicio y los metros recorridos antes y después de la intervención de cada programa de entrenamiento, basados en el MICT (Grupo 1) y HIIT (Grupo 2), con un componente de fuerza muscular, se observó una mejora significativa en todas las variables ( $p=<0,05 \%$ ). Además, al comparar los resultados entre los grupos, fue posible demostrar mejores resultados con una diferencia significativa en el grupo experimental 2 ( $p=<0,05 \%$ ) (Cuadro 2).

Durante las sesiones de los programas de entrenamiento, los participantes informaron una disnea percibida según la escala de Borg, entre 6 y 8 (intensidad moderada), en los grupos 1 y 2 , resaltando que el grupo 2 tuvo picos entre 9 y 10, así como la sensación de fatiga durante el ejercicio.

Luego de las respectivas intervenciones, se encontraron cambios significativos en la fracción de eyección del ventrículo izquierdo del grupo MICT $(51 \pm 4,5$ vs $52 \pm 5,4)$ y grupo HIIT $(45 \pm 5,3$ vs $49 \pm 3,2)$. Además, los niveles de Vo2 y MET fueron superiores en el GE1 $(8,3 \pm 3,2$ vs $13,1 \pm 5,8)$ y GE2 $(8,5 \pm 8,3$ vs $16,7 \pm 5,5)$ en comparación con el GC $(8,9 \pm 5,7$ vs $8,8 \pm 4,0)$. Al igual, la fatiga asociada al cáncer de mama en el grupo HIIT $(20,4 \pm 5,6$ vs $5,1 \pm 3,6)$ y MICT $(18,6 \pm 9,5$ vs $8,0 \pm 4,2)$. Para el grupo control no hubo mejoría $(16,6 \pm 5,5$ vs $16,9 \pm 4,6)$ (Cuadro 2). Durante los programas de intervención no se presentaron complicaciones ni eventos adversos debidos al entrenamiento físico realizado por las pacientes con cáncer de mama en estadio II.

\section{Discusión}

En un estudio realizado por Adamsen et al., ${ }^{21}$ con 269 pacientes con cáncer que realizaron ejercicio supervisado, se incluyó entrenamiento cardiovascular y de resistencia de alta intensidad, entrenamiento de relajación y conciencia corporal. Las sesiones tuvieron una duración de 9 horas semanales, durante 6 semanas, donde se determinó que la intervención redujo la fatiga y mejoró la vitalidad, capacidad aeróbica, fuerza muscular, actividad física y el bienestar emocional, pero no la calidad de vida. Al igual, Heinrich et al.22 investigaron la viabilidad y la eficacia de un programa de ejercicios en grupo de entrenamiento funcional de alta intensidad (HIFT) en 8 adultos sobrevivientes de cáncer, dentro de los 5 años del último tratamiento. Los hallazgos mostraron que hubo mejoras significativas para el estado emocional $(p=0,042)$ y la composición corporal (masa magra $p=0,008$; masa grasa $p=0,001$; porcentaje de grasa corporal $p<0,001$ ). Los participantes, también mejoraron significativamente en cinco de los siete movimientos funcionales: equilibrio $(p=0,032)$, portando un objeto ponderado $(p=0,004)$, fuerza y potencia de la parte inferior del cuerpo $(p=0,009)$, capacidad aeróbica y resistencia $(p=0,039)$, y flexibilidad $(p=0,012)$. Estos datos coinciden con nuestros resultados donde el HIIT mejoró todos los valores frente al MCIT y el grupo control.

Por otro lado, Schmitt et al. ${ }^{23}$ compararon los efectos de una rehabilitación multimodal de 3 semanas que incluye entrenamiento supervisado por intervalos de alta intensidad (HIIT) en mujeres sobrevivientes de cáncer de mama con respecto a variables clave de acondicionamiento aeróbico, composición corporal, gasto de energía, fatiga relacionada con el cáncer y calidad de vida. Schulz et al. ${ }^{24}$ evaluaron la viabilidad de una intervención de ejercicio que consiste en resistencia a intervalos de alta intensidad y entrenamiento de fuerza en 26 mujeres con cáncer de mama no metastásico. Se mostró una mejora en el pico de VO2 (12,0 $\pm 13,0 \%)$ y el rendimiento de la fuerza $(25,9 \pm 11,2 \%)$, al igual que en la presente investigación y en la de Schulz et al.

Mijwel et a ${ }^{25}$ compararon los efectos de la resistencia y el entrenamiento en intervalos de alta intensidad (RT - HIIT), y el entrenamiento aeróbico de intensidad moderada y el entrenamiento en intervalos de alta intensidad (AT - HIIT), con la atención habitual en 240 mujeres con cáncer de mama sometidas a quimioterapia; este programa de ejercicios constó de 16 semanas de entrenamiento de resistencia y HIIT. Los hallazgos determinaron que el grupo RT - HIT tuvo mejoraría sobre la capacidad funcional ( $p=0,02$ ) y las actividades de la vida diaria $(p=0,01)$. El grupo de AT-HIT mejoró significativamente el funcionamiento emocional $(p=0,01$ ) y los síntomas del dolor $(p=0,03)$. Sin embargo, el grupo de RT-HIIT mostró una disminución de síntomas, mientras que AT - HIIT se mantuvo estable $(p<0,01)$, lo que sugiere que las implicaciones del entrenamiento de la resistencia, al igual que en nuestro estudio, mejorarían los valores determinantes de la capacidad aeróbica y funcional en los pacientes con CA de mama. Otros estudios actuales 7,26 han demostrado los beneficios del entrenamiento interválico de alta intensidad con ejercicio de resistencia o fuerza en pacientes con cáncer de mama.

Ahora bien, los diferentes hallazgos expresados en la presente investigación también concuerdan claramente con el National Comprehensive Cancer Network Guidelines, que señala que el ejercicio es uno de los tratamientos no farmacológicos más efectivos para la fatiga asociada al cáncer. Sin embargo, la inclusión de programas de ejercicio en las guías actuales de rehabilitación oncológica todavía no es estándar. Nuestros resultados indican claramente que esto debe considerarse. Esto concuerda con los resultados de publicaciones de los países bajos, ${ }^{27}$ Asia, ${ }^{28,29}$ India, ${ }^{30}$ China ${ }^{31}$ y otros. ${ }^{32-39}$

\section{Conclusiones}

El entrenamiento HIIT se mostró seguro y beneficioso para las mujeres con cáncer de mama en estadio II. Mejoró la tolerancia al ejercicio, la fuerza, vo2, MET, y sobre todo, la fatiga asociada al cáncer y la calidad de vida de las pacientes. De hecho, fue posible demostrar mayores beneficios en las pacientes que recibieron HIIT en comparación con el grupo MICT y el grupo control. No obstante, el grupo de entrenamiento MICT también presentó diferencias significativas en todas las variables evaluadas. E1 


\section{Fatiga asociada al cáncer de mama / Pereira-Rodríguez et al}

grupo control de atención habitual sin entrenamiento físico ni ejercicio supervisado no presentó cambios significativos ni mejoras en la fatiga asociada al cáncer de mama.

\section{Referencias}

1. Padilha CS, Marinello PC, Galvão DA, Newton RU, Borges FH, Frajacomo F. et al. Evaluation of resistance training to improve muscular strength and body composition in cancer patients undergoing neoadjuvant and adjuvant therapy: a meta-analysis. J Cancer Surviv. 2017; 11: 339-349.

2. Mitrus I, Bryndza E, Sochanik A, \& Szala S. Evolving models of tumor origin and progression. Tumor Biology. 2012;4:911-917.

3. Malvezzi M, Cariolli G, Bertuccio P, Bofetta P, Levi F, La Vecchia C, et al. European cancer mortality predictions for the year 2017, with focus on lung cancer. Ann Oncol. 2017; 5: 1117-1123.

4. Plummer M, de Martel C, Vignat J, Ferlay J, Bray F, Franceschi S. Global burden of cancers attributable to infections in 2012: a synthetic analysis. Lancet Glob Health. 2016; 9: 609-16.

5. Barrios E. \& Garau M. Epidemiología del cancer en uruguay y el mundo. An Facultad Med. 2017;4:9-46.

6. Hayes S, Spence R, Galvão D, \& Newton RU. Australian Association for Exercise and Sport Science position stand: Optimising cancer outcomes through exercise. J Sci Med Sport. 2019; 12:428-34.

7. Torres VB, Vassalo J, Silva UV, Caruso P, Torelly AP, Silva E, et al. Outcomes in Critically Ill Patients with Cancer-Related Complications. PLOS ONE. 2016; 11: 1-14.

8. Hojman P, Gehl J, Christensen J, \& Pedersen BK. Molecular Mechanisms Linking Exercise to Cancer Prevention and Treatment. Cell Metabolism. 2018; 1: $10-21$.

9. Toohey K, Pumpa K, McKune A, Cooke J, DuBose KD, Yip D, et al. Does low volume high-intensity interval training elicit superior benefits to continuous low to moderate-intensity training in cancer survivors? World J Clin Oncol. 2018; 1: 1-12.

10. Lee K, Kang I, Mortimer J, Sattler F, Mack WJ., Fitzsimons LA, et al. Effects of high-intensity interval training on vascular function in breast cancer survivors undergoing anthracycline chemotherapy: design of a pilot study. BMJ Open. 2018; 8: 1-9.

11. Mugele H, Freitag N, Wilhelmi J, Yang Y, Cheng S, Bloch W, et al. Highintensity interval training in the therapy and aftercare of cancer patients: a systematic review with meta-analysis. J Cancer Surviv. 2019;2:205-223

12. Alansare A, Alford K, Lee S, Church T, \& Jung HC. The Effects of High-Intensity Interval Training vs. Moderate-Intensity Continuous Training on Heart Rate Variability in Physically Inactive Adults. Int J Environ Res Public Health. 2018; 7: 1-10.

13. Tew GA, Leighton D, Carpenter R, Anderson S, Langmead L, Ramage J, et al. High-intensity interval training and moderate-intensity continuous training in adults with Crohn's disease: a pilot randomised controlled trial. BMC Gastroenterol. 2019; 1: 1-11.

14. Zafirova B, \& Todorovska L. Anthropometric parameters of growth and nutritional status in children aged 6 to 7 years in R. Macedonia. Adv Med Sci. 2009; 2: 289-295.

15. Fett $\mathrm{C}$, Fett $\mathrm{W}, \&$ Marchini J. Exercício resistido vs jogging em fatores de risco metabólicos de mulheres com sobrepeso/obesas. Arq Bras Cardiol. 2009; 93 : 519-525.

16. Enright $\mathrm{P}$, \& Sherrill D. Reference Equations for the Six- Minute Walk in Healthy Adults. Am J Respir Crit Care Med. 1998; 158: 1384-1387.

17. ATS statement: guidelines for the six-minute walk test. Am J Respir Crit Care Med. 2002; 166: 111-117.

18. Yellen SB, Cella DF, Webster K, Blendowski C, \& Kaplan E. Measuring fatigue and other anemia-related symptoms with the Functional Assessment of Cancer Therapy (FACT) measurement system. J Pain Symptom Manage. 1997; 13:63-74.

19. Aaronson NK, Ahmedzai S, Bergman B, Bullinger M, Cull A, Duez NJ, et al. The European Organization for Research and Treatment of Cancer OLO-C30:
A quality-of-life instrument for use in international clinical trials in oncology. J Natl Cancer Inst. 1993; 85: 365-376.

20. Aaronson NK, Ahmedzai S, Bergman B, Bullinger M, Cull A, Duez NJ, et al. The European Organisation for Research and Treatment of Cancer OLO-C30: A quality-of-life instrument for use in international clinical trials in oncology. J Natl Cancer Inst. 1993; 85: 365-376.

21. Adamsen L, Quist M, Andersen C, Moller T, Herrstedt J, Kronborg D, et al. Effect of a multimodal high intensity exercise intervention in cancer patients undergoing chemotherapy: randomised controlled trial. BMJ. 2009; 339: 3410-3410.

22. Heinrich KM, Becker C, Carlisle T, Gilmore K, Hauser J, Frye J, et al. Highintensity functional training improves functional movement and body composition among cancer survivors: a pilot study. Eur J Cancer Care. 2015; 24: 812-817.

23. Schmitt J, Lindner N, Reuss-Borst M, Holmberg HC, \& Sperlich B. A 3-week multimodal intervention involving high-intensity interval training in female cancer survivors: a randomized controlled trial. Physiol Rep. 2016; 3: 1-8.

24. Schulz SVW, Laszlo R, Otto S, Prokopchuk D, Schumann U, Ebner F, et al. Feasibility and effects of a combined adjuvant high-intensity interval/strength training in breast cancer patients: a single-center pilot study. Disabil Rehabil. 2017; 40: 1501-1508.

25. Mijwel S, Backman M, Bolam KA, Jervaeus A, Sundberg CJ, Margolin S, et al. Adding high-intensity interval training to conventional training modalities: optimizing health-related outcomes during chemotherapy for breast cancer: the OptiTrain randomized controlled trial. Breast Cancer Res Treat. 2017;1:79-93.

26. Adams SC, De Lorey DS, Davenport MH, Fairey AS, North S, Courneya KS. Effects of high-intensity interval training on fatigue and quality of life in testicular cancer survivors. Br J Cancer. 2018; 10: 1313-1321.

27. Kessels E, Husson O, \& van der Feltz-Cornelis CM. The effect of exercise on cancer-related fatigue in cancer survivors: a systematic review and metaanalysis. Neuropsychiatric Disease and Treatment. 2018; 14: 479-494.

28. Xu Y. Intervention of aerobic exercise on the cancer-related fatigue in breast cancer patients undergoing chemotherapy in out- patient department. Hainan Med J. 2012; 19: 145-147.

29. Hu JP, Ren T, Tan BX, Gao O, \& Wu HM. Effects of aerobic exercises on cancer-related fatigue for patients with nasopharyngeal carcinoma undergoing radiotherapy. Modern Oncology. 2012; 6: 1155-1157.

30. Patel JG, \& Bhise AR. Effect of aerobic exercise on cancer-related fatigue. Indian J Palliat Care 2017; 23: 355-61.

31. Yang B, Yang B, Wang J, \& Wang J. Effects of Exercise on Cancer-related Fatigue and Quality of Life in Prostate Cancer Patients Undergoing Androgen Deprivation Therapy: A Meta-analysis of Randomized Clinical Trials. Chinese Medical Sciences Journal. 2017; 1: 13-21.

32. Bourke L, Doll H, Crank H, Daley A, Rosario D, \& Saxton JM. Lifestyle intervention in men with advanced prostate cancer receiving androgen suppression therapy: a feasibility study. Cancer Epidemiol Biomarkers Prev. 2011; 20: 647-57.

33. Bourke L, Gilbert S, Hooper R, Steed LA, Joshi M, Catto JW, et al. Lifestyle changes for improving disease-specific quality of life in sedentary men on longterm androgen-deprivation therapy for advanced prostate cancer: a randomised controlled trial. Eur Urol. 2014; 65: 865-72.

34. Repka CP, \& Hayward R. Effects of an Exercise Intervention on Cancer-Related Fatigue and Its Relationship to Markers of Oxidative Stress. Integr Cancer Ther. 2018; 2: 503-510.

35. Mustian K, Peppone L, Palesh O, Janelsins M, Mohile S, \& Purnell J. Exercise and Cancer-related Fatigue. US Oncol. 2009; 2: 20-23.

36. Kelley G, \& Kelley K. Exercise and cancer-related fatigue in adults: a systematic review of previous systematic reviews with meta-analyses. BMC Cancer. 2017; 1: $1-17$.

37. Meneses-Echavez JF., Gonzalez-Jimenez E., \& Ramirez-Velez R. Effects of supervised exercise on cancer-related fatigue in breast cancer survivors: a systematic review and meta-analysis. BMC Cancer. 2015; 1: 1-13.

38. Meneses-Echavez JF., Gonzalez-Jimenez E., \& Ramirez-Velez R. Supervised exercise reduces cancer-related fatigue: a systematic review. J Physiother. 2015; $1: 3-9$.

39. Tian L, Lu HJ, Lin L, \& Hu Y. Effects of aerobic exercise on cancer-related fatigue: a meta-analysis of randomized controlled trials. Support Care Cancer 2016; 2: 969-83. 